\title{
Histiocytofibrome bénin du plancher buccal : présentation d'un cas
}

\author{
Bassima Chami, Bouchra Taleb, Wafae EI Wady \\ Service d'Odontologie chirurgicale, Faculté de Médecine dentaire, Rabat, Maroc \\ bassima.chami1@gmail.com
}

L'histiocytofibrome bénin est une tumeur mésenchymateuse composée de fibroblastes et d'histiocytes.

Cette lésion appartient aux tumeurs dites fibrohistiocytaires qui comportent un large spectre de lésions allant de lésions dites réactionnelles comme le xantome à l'histiocytofibrome bénin qualifié de véritable tumeur.

Un patient âgé de 50 ans est venu consulter pour une tuméfaction située sur le plancher buccal droit. La tuméfaction a évolué de façon asymptomatique depuis trois ans. L'examen exo- buccal était sans particularités. L'examen endo-buccal a montré une masse circonscrite, indolore, recouverte d'une muqueuse d'aspect normal. La radiographie panoramique l'échographie n'ont pas été contributives. Ce tableau clinique faisait évoquer une tumeur bénigne de la muqueuse du plancher.

L'exérèse chirurgicale de la lésion a été réalisée sous anesthésie locale.

L'examen anatomopathologique a montré qu'il s'agissait d'un histiocytofibrome bénin.

Les examens en immunohistochimie ont confirmé ce diagnostic.

L'histiocytofibrome bénin est une lésion souvent décrite sur le revêtement cutané où il prend le nom de dermatofibrome.

Pour Skoulakis et al. (2007), il représente 1\% de l'ensemble des lésions fibrohistiocytaires, 15\% des tumeurs des tissus mous et moins de 5\% de l'ensemble des tumeurs cervico-faciales.

Selon Giovani et al. (2010), l'histiocytofibrome bénin de localisation buccale peut siéger sur la langue, des lèvres, le palais mou, le plancher buccal ou les maxillaires.

Son étiopathogénie n'est pas connue.

Cliniquement il se présente sous la forme d'un nodule asymptomatique, de croissance lente.

L'exérèse chirurgicale constitue le traitement habituel ; les récidives sont rares.

This is an Open Access article distributed under the terms of the Creative Commons Attribution License 2.0, which permits unrestricted use, distribution, and reproduction in any medium, provided the original work is properly cited. 